\title{
Improved Fully Automatic Liver Segmentation Using Histogram Tail Threshold Algorithms
}

\author{
Kyung-Sik Seo \\ Dept. of Electrical \& Computer Engineering, \\ New Mexico State University, Las Cruces, NM, USA \\ nmsu2@hanmail.net
}

\begin{abstract}
In order to remove neighboring abdominal organs of the liver, we propose an improved fully automatic liver segmentation using histogram tail threshold (HTT) algorithms. A region of interest of the liver is first segmented. A left HTT (LHTT) algorithm is performed to eliminate the pancreas, spleen, and left kidney. After the right kidney is eliminated by the right HTT (RHTT) algorithm, the robust liver structure is segmented. From the results of experiments, the improved automatic liver segmentation using HTT algorithms has strong similarity performance as manual segmentation by medical doctor.
\end{abstract}

\section{Introduction}

A computed tomography (CT) is currently a conventional and excellent tool for diagnosis of the liver in medical imaging technology. Liver segmentation using the CT has been performed often [1, 2]. As previous researches have depended on semiautomatic liver segmentation such as a seed point, a rand mark, a reference image, and training data, fully automatic liver segmentation based on the spine was proposed [3]. However, proposed liver segmentation has problems because of pixel similarity of neighboring abdominal organs such as the spleen, pancreas, and kidneys.

In order to segment neighboring organs, we propose improved fully automatic liver segmentation using histogram tail threshold (HTT) algorithms. The region of interest (ROI) of the liver structure is first segmented. Then liver segmentation using HTT algorithms are processed to eliminate neighboring abdominal organs.

\section{Improved Liver Segmentation}

As pre-processing, the ROI of the liver is extracted using multi-modal threshold [3-5], C-class maximum a posteriori [6], and binary morphological (BM) filtering [7-9]. Figure 1 shows the ROI extraction of the liver.

The left HTT (LHTT) is presented to remove neighboring abdominal organs such as the pancreas, spleen, and left kidney from the ROI. Let $I_{R O I}(m, n)$ be the graylevel ROI and $h_{R O I}\left(k_{1}, k_{2}\right)$ be the histogram of $I_{R O I}$ with the range, $\left[k_{1}, k_{2}\right]$. Let $I_{\text {LHTT }}$ be the LHTT image. Then the LHTT algorithm is proposed: 


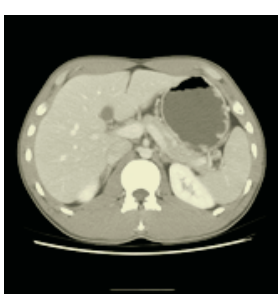

(a)

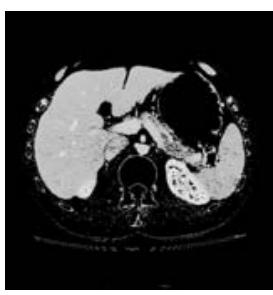

(b)

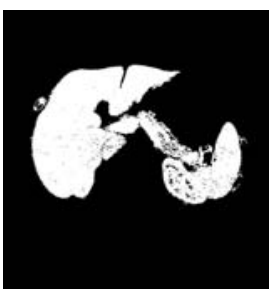

(c)

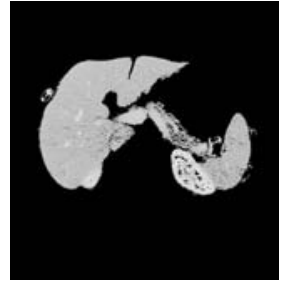

(d)

Fig. 1. ROI extraction of the liver: (a) CT image, (b) ROI after MMT, (c) ROI after C-class MAP decision, (d) Gray-level ROI

- Find $k_{\max }$ where $k_{\max }$ is the gray-level value when $h_{R O I}(k)$ is the maximum value.

- Calculate the left histogram tail interval $k_{L I}=\left(k_{\max }-k_{1}\right)$.

- $\quad$ Find the left histogram tail threshold value.

- $\quad$ Create the LHTT image $I_{\text {LHTT }}=\left\{(m, n) \mid k_{1} \leq I_{R O I}(m, n) \leq k_{L H T T}\right\}$.

Figure 2 shows an example of the LHTT algorithm.

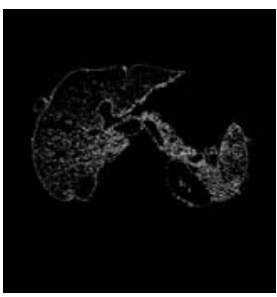

(a)

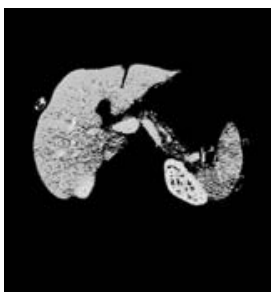

(b)

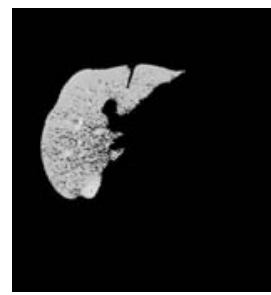

(c)

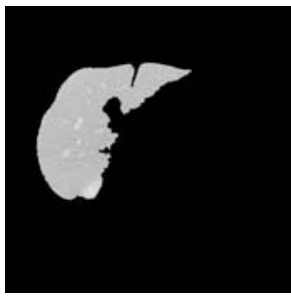

(d)

Fig. 2. Left histogram tail threshold: (a) LHTT image, (b) Difference image between $I_{R O I}$ and $I_{L H T T}$, (c) Segmented liver structure by area estimation and C-class MAP decision, (d) Robust liver segmentation by BM filtering and gray-level transformation

As the right kidney is adjacent to the lower liver part and has the same gray-level values as liver vessels, this kidney creates problems in segmenting a liver structure. The RHTT is presented for extracting and removing the right kidney from the liver image. Let $I_{R H T T}$ be the RHTT image. Then the RHTT algorithm is proposed:

- Find $k_{\max }$ where $k_{\max }$ is the gray-level value when $h_{R O I}(k)$ is the maximum value.

- $\quad$ Calculate the right partial histogram interval $k_{R I}=\left(255-k_{\max }\right)$. 
- $\quad$ Find the right partial threshold value $k_{R H T T}=\left(k_{\max }+k_{R I} / 8\right)$.

- $\quad$ Create the RHTT image $I_{R H T T}=\left\{(m, n) \mid k_{R H T T} \leq I_{R O I}(m, n) \leq 255\right\}$.

Figure 3 shows an example of the RHTT algorithm.

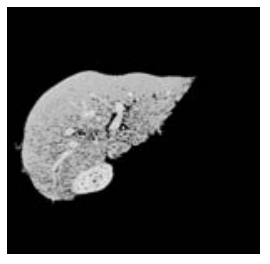

(a)

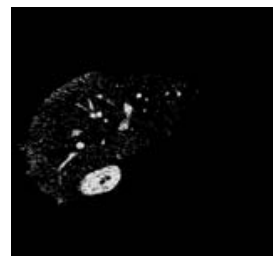

(b)

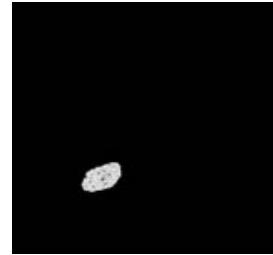

(c)

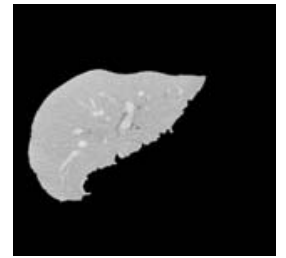

(d)

Fig. 3. Example of right histogram tail threshold: (a) Sample image, (b) RHTT image, (c) Extraction of right kidney after the area and angle estimation, (d) Elimination of the right kidney

\section{Experiments and Analysis}

40 slices of eight patients were selected for testing the improved segmentation method and one medical doctor in Chonnam National University Hospital segmented the liver structure by the manual method. In order to evaluate performance of the improved algorithm, three different segmentation methods were compared by using normalized average area (NAA) with the image size and area error rate (AER) [3]. Figure 4(a) shows NAA using error bars. Average NAAs of each method were 0.1972, 0.1593, and 0.1644. Also, Figure 4(b) shows the comparison of average AER per each patient based on manual segmentation to segmentation before and after HTT. The former is $4 \sim 40 \%$ and the latter is 5 10\%. Total average AER for all patients is $23.9198 \%$ for the former and $7.4223 \%$ for the latter. From the results of this comparison, automatic segmentation using HTT algorithms matches the results of manual segmentation more closely than segmentation without HTT algorithms.

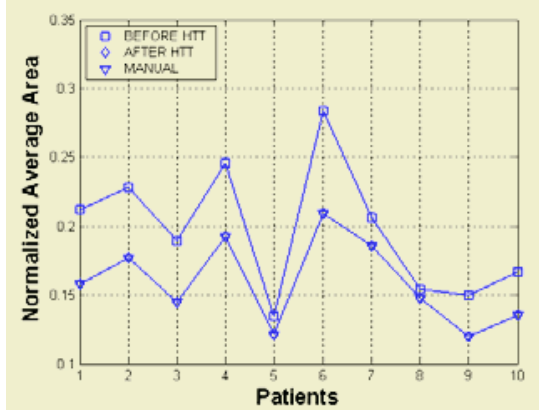

(a)

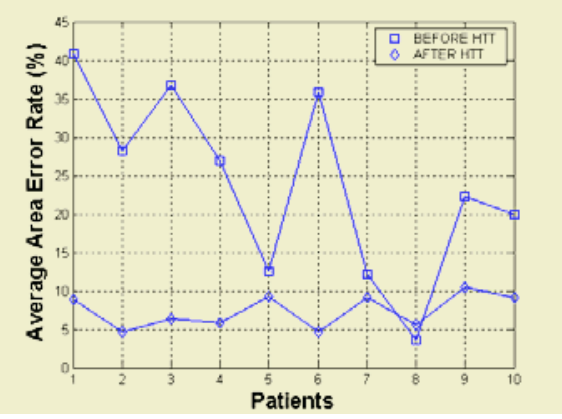

(b)

Fig. 4. Results: (a) Normalized average area, (b) Average area error rate 


\section{Conclusions}

In this paper, we proposed the improved fully automatic liver segmentation using histogram tail threshold (HTT) algorithms. 40 slices of eight patients were selected to evaluate performance of the improved algorithm. From the results of experiments, the improved automatic liver segmentation has strong similarity performance as manual segmentation by medical doctor.

\section{References}

1. Bae, K. T., Giger, M. L., Chen, C. T., Kahn, Jr. C. E.: Automatic segmentation of liver structure in CT images. Med. Phys.,Vol. 20. (1993) 71-78

2. Gao, L., Heath, D. G., Kuszyk, B. S., Fishman, E. K.: Automatic liver segmentation technique for three-dimensional visualization of CT data. Radiology, Vol. 201. (1996) 359-364

3. 3.Seo, K., Ludeman, L. C., Park S., Park, J.: Efficient liver segmentation based on the spine. LNCS, Vol. 3261. (2004) 400-409.

4. 4.Orfanidis, S. J.: Introduction to signal processing. Prentice Hall, Upper Saddle River NJ (1996)

5. 5.Schilling, R. J., Harris, S. L.: Applied numerical methods for engineers. Brooks/Cole Publishing Com., Pacific Grove CA (2000)

6. Ludeman, L. C.: Random processes: filtering, estimation, and detection. Wiley \& Sons Inc., Hoboken NJ (2003)

7. Gonzalez, R. C., Woods, R. E.: Digital image processing. Prentice Hall, Upper Saddle River NJ (2002)

8. Shapiro, L. G., Stockman, G. C.: Computer vision. Prentice-Hall, Upper Saddle River NJ (2001)

9. Parker, J.R.: Algorithms for image processing and computer vision. Wiley Computer Publishing, New York (1997) 